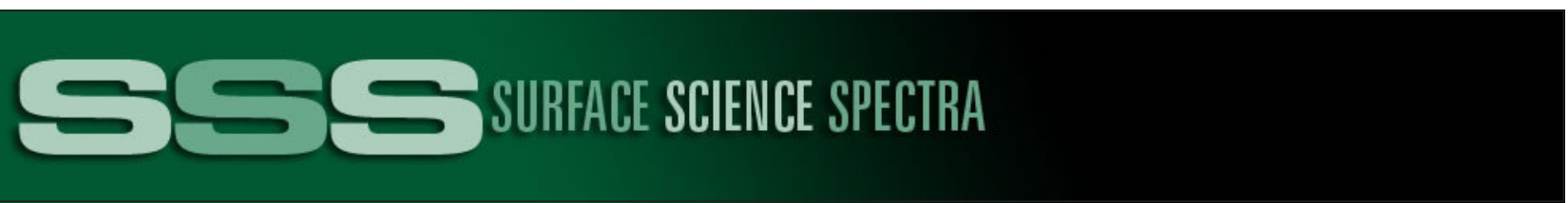

\title{
Enargite by XPS
}

Marzia Fantauzzi, Davide Atzei, Stefania Da Pelo, Bernhard Elsener, Franco Frau et al.

Citation: Surf. Sci. Spectra 9, 266 (2002); doi: 10.1116/11.20030801

View online: http://dx.doi.org/10.1116/11.20030801

View Table of Contents: http://avspublications.org/resource/1/SSSPEN/v9/i1

Published by the AVS: Science \& Technology of Materials, Interfaces, and Processing

\section{Related Articles}

X-ray photoelectron spectroscopy study on the chemistry involved in tin oxide film growth during chemical vapor deposition processes

J. Vac. Sci. Technol. A 31, 01A105 (2013)

High-temperature stability of postgrowth-annealed Al-doped MgxZn1-xO films without the phase separation effect J. Vac. Sci. Technol. B 30, 061201 (2012)

$\mathrm{Cl}$ atom recombination on silicon oxy-chloride layers deposited on chamber walls in chlorine-oxygen plasmas J. Vac. Sci. Technol. A 30, 051307 (2012)

Interactions of chlorine plasmas with silicon chloride-coated reactor walls during and after silicon etching J. Vac. Sci. Technol. A 30, 051306 (2012)

$\mathrm{Pt}(\mathrm{II})$ and $\mathrm{Pd}(\mathrm{II})$ Pyrrolidine-Dithiocarbamates Investigated by XPS

Surf. Sci. Spectra 18, 82 (2011)

\section{Additional information on Surf. Sci. Spectra}

Journal Homepage: http://avspublications.org/sss

Journal Information: http://avspublications.org/sss/about/about_the_journal

Top downloads: http://avspublications.org/sss/top_20_most_downloaded

Information for Authors: http://avspublications.org/sss/authors/information_for_contributors

\section{ADVERTISEMENT}

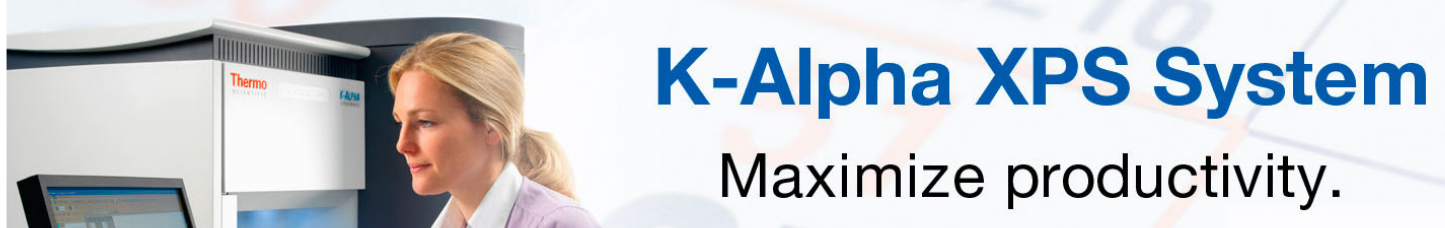




\section{Enargite by XPS}

Marzia Fantauzzi and Davide Atzei

Department of Inorganic and Analytical Chemistry, University of Cagliari, Cittadella Universitaria Monserrato, Cagliari 09100, Italy

Stefania Da Pelo

Department of Earth Sciences, University of Cagliari, via Trentino 51, Cagliari 09127, Italy

Bernhard Elsener

Deptartment of Inorganic and Analytical Chemistry, University of Cagliari, Cittadella Universitaria Monserrato, Cagliari 09100, Italy

Franco Frau and Piero Franco Lattanzi

Department of Earth Sciences, University of Cagliari, via Trentino 51, Cagliari 09127, Italy

Antonella Rossi

Department of Inorganic and Analytical Chemistry, University of Cagliari, Cittadella Universitaria di Monserrato, Cagliari, Cagliari 09100, Italy

(Received 4 August 2003; accepted 16 January 2004; published 12 March 2004)

X-ray photoelectron spectroscopy was used for characterizing the enargite surface. Freshly cleaved samples were analyzed at liquid nitrogen temperature. Enargite is a copper arsenic sulfide of formula $\mathrm{Cu}_{3} \mathrm{AsS}_{4}$; it is used as a minor ore of copper. Enargite is a potential source of arsenic and may create environmental problems through the release of toxic elements upon oxidation. (C) 2004 American Vacuum Society. [DOI: 10.1116/11.20030801]

Keywords: $x$-ray photoelectron spectroscopy; Enargite

PACS: $82.80 . P v, 91.60 .-x$

\section{SPECIMEN DESCRIPTION (Accession \#00782)}

Host Material: Natural enargite $\left(\mathrm{Cu}_{3} \mathrm{AsS}_{4}\right)$

Host Material Characteristics: homogeneous; solid; unknown crystallinity; semiconductor; inorganic compound

Chemical Name: copper arsenic sulfide

Host Composition: $\mathrm{Cu}_{3} \mathrm{AsS}_{4}$

Form: crystal

History \& Significance: The sample is a mineral extracted from mines in Furtei (CA, Italy).

As Received Condition: The sample was received as crystal.

Analyzed Region: flat surface in the "as received" condition (Refs. 1 and 2)

Ex Situ Preparation/Mounting: The sample was mounted as crystal on double sided adhesive tape.

In Situ Preparation: none

Charge Control: Sample charging was constant during analysis. Energy shift was compensated by referencing all the spectra to a C $1 s$ signal taken at $285.0 \mathrm{eV}$.

Temp. During Analysis: $77.4 \mathrm{~K}$

Pressure During Analysis: $<5 \times 10^{-8} \mathrm{~Pa}$

\section{SPECIMEN DESCRIPTION (Accession \#00783)}

Host Material: Synthetic enargite

Host Material Characteristics: homogeneous; solid; unknown crystallinity; semiconductor; inorganic compound
Accession \#s 00782, 00783

Technique: XPS

Host Material: \#00782: Natural enargite $\left(\mathrm{Cu}_{3} \mathrm{AsS}_{4}\right)$; \#00783:

Synthetic enargite

Instrument: Vacuum Generators Ltd, East Greenstead, UK Escalab Mark II with upgrade to Escalab 200

Major Elements in Spectrum: $\mathrm{Cu}$, As, S, C, O

Minor Elements in Spectrum: none Printed Spectra: 8

Spectra in Electronic Record: 20 Spectral Category: comparison 


\section{INSTRUMENT PARAMETERS COMMON TO ALL SPECTRA}

\section{Spectrometer}

Analyzer Mode: constant pass energy

Throughput $\left(T=E^{N}\right): N=-0.5$

Excitation Source Window: Al window, foil thickness $1.0 \mu \mathrm{m}$

Excitation Source: $\mathrm{Al} K_{\alpha}$

Source Energy: $1486.6 \mathrm{eV}$

Source Strength: $300 \mathrm{~W}$

Source Beam Size: $12.5 \mathrm{~mm} \times 12.5 \mathrm{~mm}$

Analyzer Width: $2000 \mu \mathrm{m} \times 5000 \mu \mathrm{m}$

Signal Mode: V/F analog

Number of Scans: 1

- Geometry

Incident Angle: $49^{\circ}$

Source to Analyzer Angle: $49^{\circ}$

Emission Angle: $0^{\circ}$

Specimen Azimuthal Angle: $40^{\circ}$

Acceptance Angle from Analyzer Axis: $0^{\circ}$

Analyzer Angular Acceptance Width: $4^{\circ} \times 8^{\circ}$ at $886 \mathrm{eV}$

Ion Gun

Manufacturer and Model: VG scientific AG 21

Energy: $4000 \mathrm{eV}$

Current: 0.180

Current Measurement Method: biased stage

Sputtering Species: Ar

Incident Angle: $48^{\circ}$

Polar Angle: $53^{\circ}$

Azimuthal Angle: $85^{\circ}$
Comment: The analyzed area was etched to remove contamination and the oxide layer. Sputtering was performed by backfilling the chamber.

\section{DATA ANALYSIS METHOD}

Energy Scale Correction: Calibration of the energy scale was performed according to Ref. 3. For charging correction, C $1 \mathrm{~s}$ was taken at $285.00 \mathrm{eV}$.

Recommended Energy-Scale Shift: For all Accession \#00782, $-1.4 \mathrm{eV}$; for all Accession \#00783, $-3.6 \mathrm{eV}$

Peak Shape and Background Method: Shirley-Sherwood background subtraction, line shape mixed product GaussianLorentzian functions with exponential tails in some cases

Quantitation Method: Surface analysis was based on the areas of the photoelectron peaks using the following equation: $C_{i}=$ $\left(I_{i} / S_{i}\right)\left[\Sigma_{i}\left(I_{i} / S_{i}\right)\right]^{-1}$, where $C_{i}$ is the atomic percentage of element $i, I_{i}$ is the intensity of the photoelectron signal (i.e., the peak area) after subtraction of a nonlinear background, and $\mathrm{Si}$ is the atomic sensitivity calculated using the photoionization cross section of Scofield (Ref. 4), corrected for the angular asymmetry function and for the asymmetry parameter (Ref. 5).

\section{REFERENCES}

1. D. Atzei, S. Da Pelo, B. Elsener, M. Fantauzzi, F. Frau, P. Lattanzi, and A. Rossi, An. Chim. 93, 11 (2003).

2. A. Rossi, D. Atzei, S. Da Pelo, F. Frau, P. Lattanzi, K. E. R. England, and D. J. Vaughan, Surf. Interface Anal. 31, 465 (2001).

3. ISO 15472: Surface Chemical Analysis - X-ray Photoelectron Spectrometers - Calibration of Energy Scales, M. P. Seah, Surf. Interface Anal. 31, 721 (2001).

4. J. H. Scofield, J. Electron Spectrosc. Relat. Phenom. 8, 129 (1976).

5. R. F. Reilman, A. Msezane, and S. T. Manston, J. Electron Spectrosc. 8, 389 (1976).

\begin{tabular}{|c|c|c|c|c|c|c|c|}
\hline \multicolumn{8}{|c|}{ SPECTRAL FEATURES TABLE } \\
\hline $\begin{array}{l}\text { Spectrum } \\
\text { ID \# }\end{array}$ & $\begin{array}{l}\text { Element/ } \\
\text { Transition }\end{array}$ & $\begin{array}{c}\text { Peak } \\
\text { Energy } \\
\text { (eV) }\end{array}$ & $\begin{array}{l}\text { Peak Width } \\
\text { FWHM } \\
(\mathrm{eV})\end{array}$ & $\begin{array}{c}\text { Peak Area } \\
\text { (eV-cts/s) }\end{array}$ & $\begin{array}{c}\text { Sensitivity } \\
\text { Factor }\end{array}$ & $\begin{array}{l}\text { Concen- } \\
\text { tration } \\
\text { (at. \%) }\end{array}$ & $\begin{array}{c}\text { Peak } \\
\text { Assignment }\end{array}$ \\
\hline $00782-02^{\mathrm{a}}$ & $\mathrm{Cu} 2 p_{3 / 2}$ & 932.2 & 1.44 & 32183 & 1.44 & 28 & $\ldots$ \\
\hline $00782-03^{\mathrm{a}}$ & As $3 d$ & 43.5 & 1.63 & 1590 & 0.17 & 12.0 & $\ldots$ \\
\hline $00782-04^{\mathrm{a}}$ & $\mathrm{S} 2 p$ & 162.1 & 2.10 & 7082 & 0.15 & 60 & $\ldots$ \\
\hline $00783-02^{\mathrm{b}}$ & $\mathrm{Cu} 2 p_{3 / 2}$ & 932.7 & 1.43 & 46068 & 1.44 & 33 & $\ldots$ \\
\hline $00783-03^{\mathrm{b}}$ & As $3 d$ & 43.5 & 1.64 & 1855 & 0.17 & 12 & $\ldots$ \\
\hline $00783-04^{b}$ & $\mathrm{~S} 2 p$ & 162.6 & 2.44 & 7929 & 0.15 & 55 & $\ldots$ \\
\hline
\end{tabular}

\footnotetext{
${ }^{a}$ Natural Enargite
}

${ }^{b}$ Synthetic Enargite

Comment to Spectral Features Table: The composition listed in item I-6 does not include carbon and oxygen because the concentration is calculated taking into account the attenuation of the emitted electrons due to the presence of the outermost layer constituted of $\mathrm{C}$ and $\mathrm{O}$. $\mathrm{C}$ and $\mathrm{O}$ do not belong to enargite but only to the surface contamination of the sample. Including $\mathrm{C}$ and $\mathrm{O}$ in the calculation would imply the wrong assumption of homogeneity of the sample.

Footnote to Spectrum 00783-04: Two $s 2 p$ signals were revealed in detailed sulfur spectra. The more intense signal at $162.5 \mathrm{eV}$ can be assigned to sulfur in the sulfide chemical state (formal oxidation state -2 ). The weak signal at $164.3 \mathrm{eV}$ was assigned to a species with a higher oxidation state. 


\begin{tabular}{|c|c|c|c|c|c|c|c|}
\hline \multicolumn{8}{|c|}{ ANALYZER CALIBRATION TABLE } \\
\hline $\begin{array}{l}\text { Spectrum } \\
\text { ID \# }\end{array}$ & $\begin{array}{l}\text { Element/ } \\
\text { Transition }\end{array}$ & $\begin{array}{c}\text { Peak } \\
\text { Energy } \\
(\mathrm{eV})\end{array}$ & $\begin{array}{c}\text { Peak Width } \\
\text { FWHM } \\
(\mathrm{eV})\end{array}$ & $\begin{array}{c}\text { Peak Area } \\
(\text { eV-cts/s) }\end{array}$ & $\begin{array}{c}\text { Sensitivity } \\
\text { Factor }\end{array}$ & $\begin{array}{l}\text { Concen- } \\
\text { tration } \\
\text { (at. \%) }\end{array}$ & $\begin{array}{c}\text { Peak } \\
\text { Assignmen }\end{array}$ \\
\hline 00786-01 & $\operatorname{Ag~} 3 d_{5 / 2}$ & 368.10 & 1.10 & 1341501 & $\cdots$ & $\cdots$ & $\cdots$ \\
\hline 00784-01 & $\mathrm{Au} 4 f_{7 / 2}$ & 83.93 & 1.28 & 982980 & $\cdots$ & $\cdots$ & $\cdots$ \\
\hline 00785-01 & $\mathrm{Cu} 2 p_{3 / 2}$ & 932.67 & 1.41 & 2426632 & $\cdots$ & $\cdots$ & $\cdots$ \\
\hline 00785-02 & $\mathrm{Cu} L M M$ & 567.96 & 1.16 & 380987 & $\cdots$ & $\cdots$ & $\cdots$ \\
\hline
\end{tabular}

\begin{tabular}{|c|c|c|c|c|c|}
\hline \multicolumn{6}{|c|}{ GUIDE TO FIGURES } \\
\hline $\begin{array}{l}\text { Spectrum } \\
\text { (Accession) \# }\end{array}$ & $\begin{array}{l}\text { Spectral } \\
\text { Region }\end{array}$ & $\begin{array}{l}\text { Voltage } \\
\text { Shift* }\end{array}$ & Multiplier & Baseline & Comment \# \\
\hline $782-1$ & Survey & +1.4 & 1 & 0 & \\
\hline $782-2$ & $\mathrm{Cu} 2 p$ & +1.4 & 1 & 0 & \\
\hline $782-3$ & As $3 d$ & +1.4 & 1 & 0 & \\
\hline $782-4$ & $\mathrm{~S} 2 p$ & +1.4 & 1 & 0 & \\
\hline $783-1$ & Survey & +3.6 & 1 & 0 & \\
\hline $783-2$ & $\mathrm{Cu} 2 p$ & +3.6 & 1 & 0 & \\
\hline $783-3$ & As $3 d$ & +3.6 & 1 & 0 & \\
\hline $783-4$ & S $2 p$ & +3.6 & 1 & 0 & \\
\hline $782-5[\mathrm{NP}]^{* *}$ & $\mathrm{Cu} L M M$ & +1.4 & 1 & 0 & \\
\hline $782-6$ [NP] & As $L M M$ & +1.4 & 1 & 0 & \\
\hline $782-7$ [NP] & $\mathrm{C} 1 s$ & +1.4 & 1 & 0 & \\
\hline $782-8$ [NP] & $\mathrm{O} 1 s$ & +1.4 & 1 & 0 & \\
\hline $783-5$ [NP] & $\mathrm{Cu} L M M$ & +3.6 & 1 & 0 & \\
\hline 783-6 [NP] & As $L M M$ & +3.6 & 1 & 0 & \\
\hline 783-7 [NP] & $\mathrm{C} 1 s$ & +3.6 & 1 & 0 & \\
\hline 783-8 [NP] & $\mathrm{O} 1 s$ & +3.6 & 1 & 0 & \\
\hline $784-1$ [NP] & $\mathrm{Au} 4 f$ & 0 & 1 & 0 & 1 \\
\hline $785-1$ [NP] & $\mathrm{Cu} 2 p$ & 0 & 1 & 0 & 1 \\
\hline $785-2$ [NP] & $\mathrm{Cu} L M M$ & 0 & 1 & 0 & 1 \\
\hline $786-1$ [NP] & $\operatorname{Ag~} 3 d$ & 0 & 1 & 0 & 1 \\
\hline
\end{tabular}

* Voltage shift of the archived (as-measured) spectrum relative to the printed figure. The figure reflects the recommended energy scale correction due to a calibration correction, sample charging, flood gun, or other phenomenon.

** [NP] signifies not published; digital spectra are archived in SSS database but not reproduced in the printed journal.

1. Calibration spectrum 


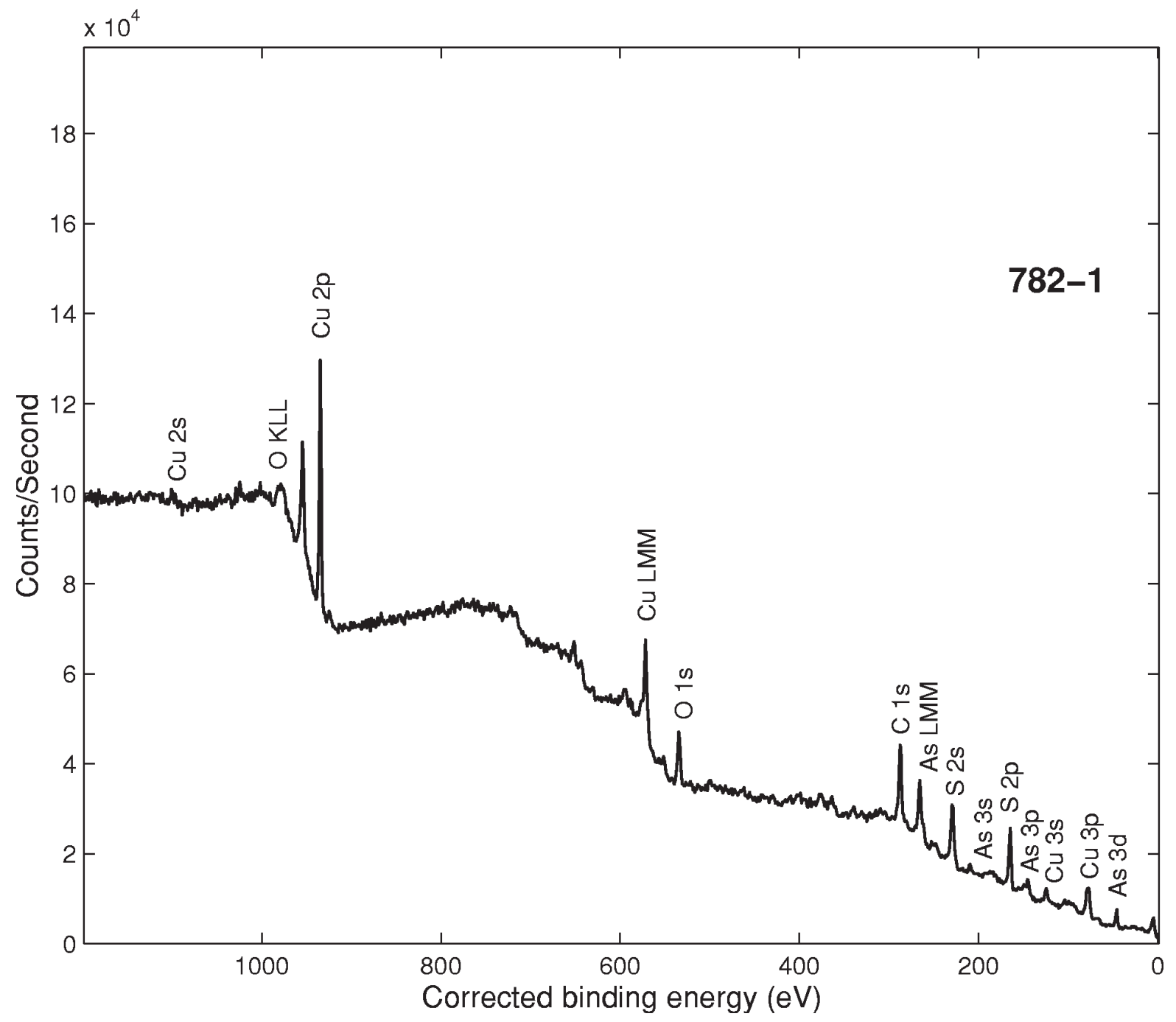

\begin{tabular}{rc}
\hline Accession \# & $\mathbf{0 0 7 8 2 - 0 1}$ \\
\hline Host Material & Natural enargite $\left(\mathrm{Cu}_{3} \mathrm{AsS}_{4}\right)$ \\
Technique & XPS \\
Spectral Region & survey \\
Instrument & Vacuum Generators Ltd,. East Greenstead, UK \\
Excitation Source & Escalab Mark II with upgrade to Escalab 200 \\
Source Energy & Al $K_{\alpha}$ \\
Source Strength & $1486.6 \mathrm{eV}$ \\
Source Size & $300 \mathrm{~W}$ \\
Analyzer Type & $12.5 \mathrm{~mm} \times 12.5 \mathrm{~mm}$ \\
Incident Angle & spherical sector \\
Emission Angle & $49^{\circ}$ \\
Analyzer Pass Energy & $0^{\circ}$ \\
Analyzer Resolution & $50 \mathrm{eV}$ \\
Total Elapsed Time & $1.12 \mathrm{eV}$ \\
Number of Scans & $140.10 \mathrm{~s}$ \\
Effective Detector Width & $140.10 \mathrm{~s}$ \\
\hline Sotal & 1 \\
\hline
\end{tabular}





- Accession \#: 00782-02

- Host Material: Natural enargite $\left(\mathrm{Cu}_{3} \mathrm{AsS}_{4}\right)$

- Technique: XPS

- Spectral Region: Cu $2 p$

Instrument: Vacuum Generators Ltd., East Greenstead, UK Escalab Mark II with upgrade to Escalab 200

Excitation Source: Al $K_{\alpha}$ Source Energy: $1486.6 \mathrm{eV}$ Source Strength: $300 \mathrm{~W}$ Source Size: $12.5 \mathrm{~mm} \times 12.5 \mathrm{~mm}$ Incident Angle: $49^{\circ}$ Analyzer Type: spherical sector Analyzer Pass Energy: $20 \mathrm{eV}$ Analyzer Resolution: $1.12 \mathrm{eV}$ Emission Angle: $0^{\circ}$ Total Signal Accumulation Time: $90.10 \mathrm{~s}$

Total Elapsed Time: $90.10 \mathrm{~s}$

Number of Scans: 1

Effective Detector Width: $0.04 \mathrm{eV}$

口 Accession \#: 00782-03

- Host Material: Natural enargite $\left(\mathrm{Cu}_{3} \mathrm{AsS}_{4}\right)$

- Technique: XPS

- Spectral Region: As $3 d$

Instrument: Vacuum Generators Ltd., East Greenstead, UK Escalab Mark II with upgrade to Escalab 200

Excitation Source: $\mathrm{Al} \mathrm{K}_{\alpha}$ Source Energy: $1486.6 \mathrm{eV}$ Source Strength: $300 \mathrm{~W}$ Source Size: $12.5 \mathrm{~mm} \times 12.5 \mathrm{~mm}$ Incident Angle: $49^{\circ}$ Analyzer Type: spherical sector Analyzer Pass Energy: $20 \mathrm{eV}$ Analyzer Resolution: $1.12 \mathrm{eV}$ Emission Angle: $0^{\circ}$

Total Signal Accumulation Time: $150.30 \mathrm{~s}$

Total Elapsed Time: $150.30 \mathrm{~s}$

Number of Scans: 3

Effective Detector Width: $0.4 \mathrm{eV}$ 


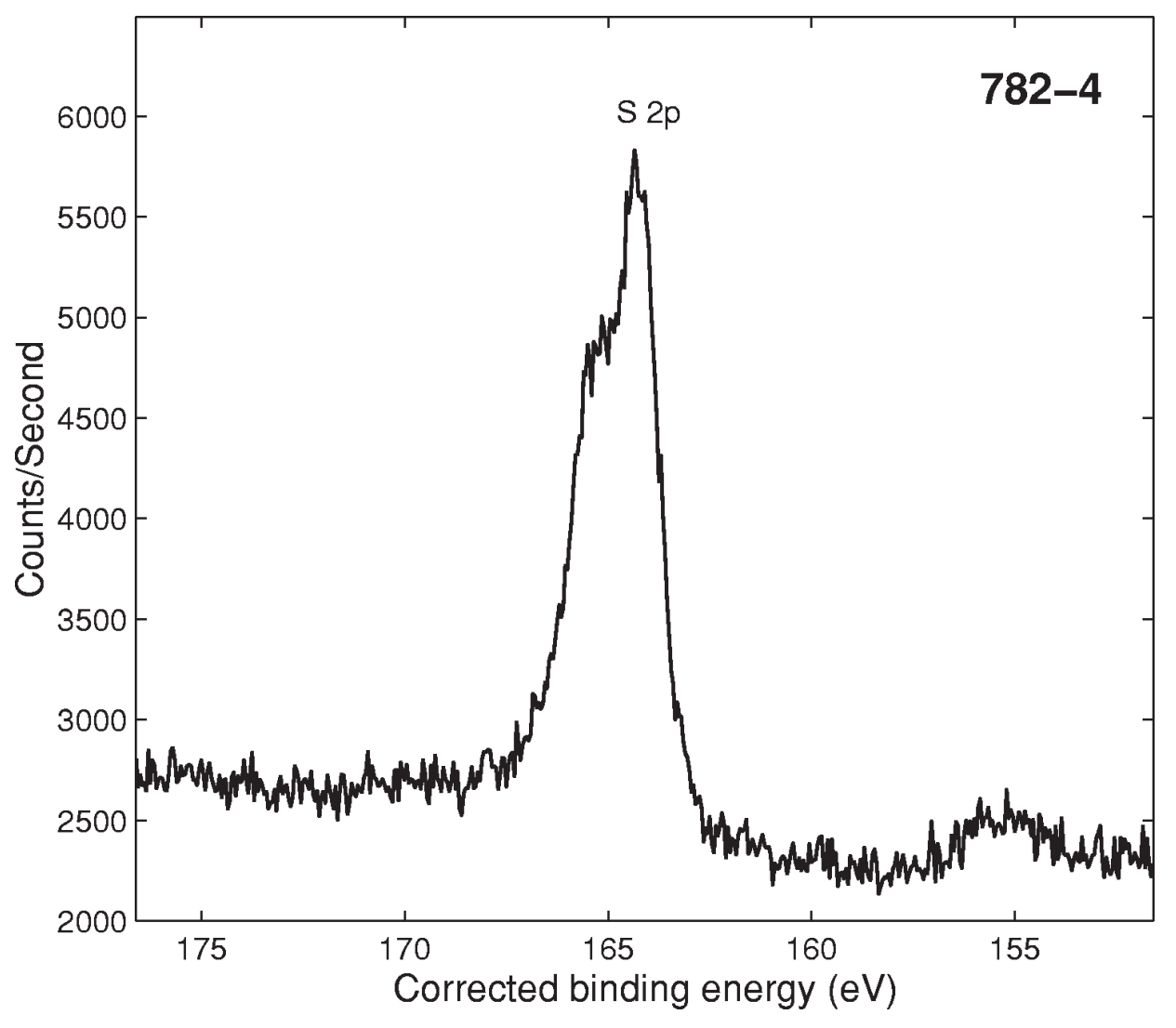

- Accession \#: 00782-04

- Host Material: Natural enargite $\left(\mathrm{Cu}_{3} \mathrm{AsS}_{4}\right)$

- Technique: XPS

— Spectral Region: $S 2 p$

Instrument: Vacuum Generators Ltd., East Greenstead, UK Escalab Mark II with upgrade to Escalab 200

Excitation Source: $\mathrm{Al} K_{\alpha}$ Source Energy: $1486.6 \mathrm{eV}$ Source Strength: $300 \mathrm{~W}$

Source Size: $12.5 \mathrm{~mm} \times 12.5 \mathrm{~mm}$ Incident Angle: $49^{\circ}$ Analyzer Type: spherical sector Analyzer Pass Energy: $20 \mathrm{eV}$ Analyzer Resolution: $1.12 \mathrm{eV}$ Emission Angle: $0^{\circ}$

Total Signal Accumulation Time: $150.30 \mathrm{~s}$

Total Elapsed Time: $150.30 \mathrm{~s}$

Number of Scans: 3

Effective Detector Width: $0.4 \mathrm{eV}$ 


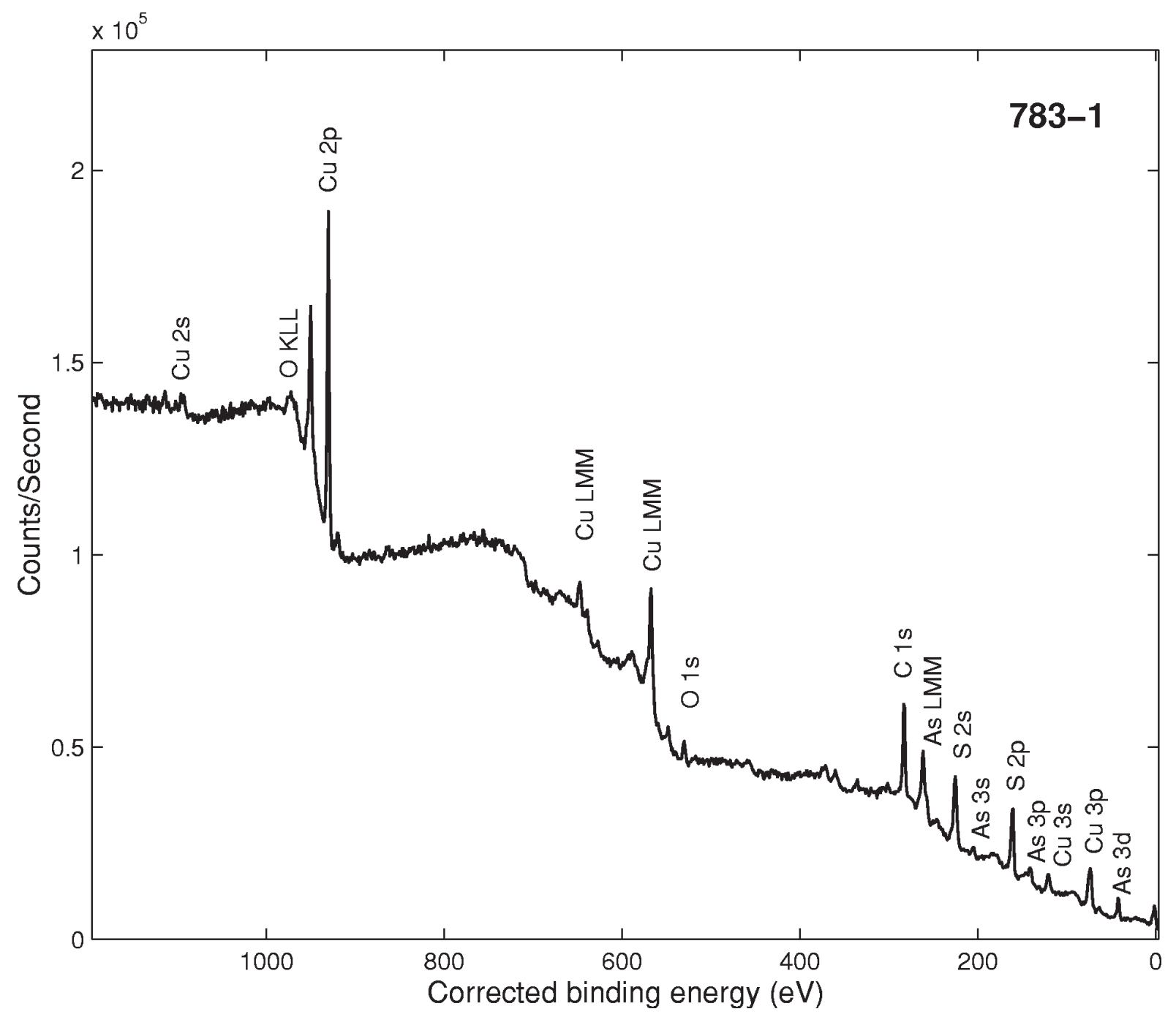

\begin{tabular}{rc}
\hline Accession \# & 00783-01 \\
\hline Host Material & Synthetic enargite \\
Technique & XPS \\
Spectral Region & survey \\
Instrument & Vacuum Generators Ltd., East Greenstead, UK \\
Excitation Source & Escalab Mark II with upgrade to Escalab 200 \\
Source Energy & Al $K_{\alpha}$ \\
Source Strength & $1486.6 \mathrm{eV}$ \\
Source Size & $300 \mathrm{~W}$ \\
Analyzer Type & $12.5 \mathrm{~mm} \times 12.5 \mathrm{~mm}$ \\
Incident Angle & spherical sector \\
Emission Angle & $49^{\circ}$ \\
Analyzer Pass Energy & $0^{\circ}$ \\
Analyzer Resolution & $50 \mathrm{eV}$ \\
Total Elapsed Time & $1.12 \mathrm{eV}$ \\
Number of Scans & $140.10 \mathrm{~s}$ \\
Effective Detector Width & $140.10 \mathrm{~s}$ \\
\hline
\end{tabular}




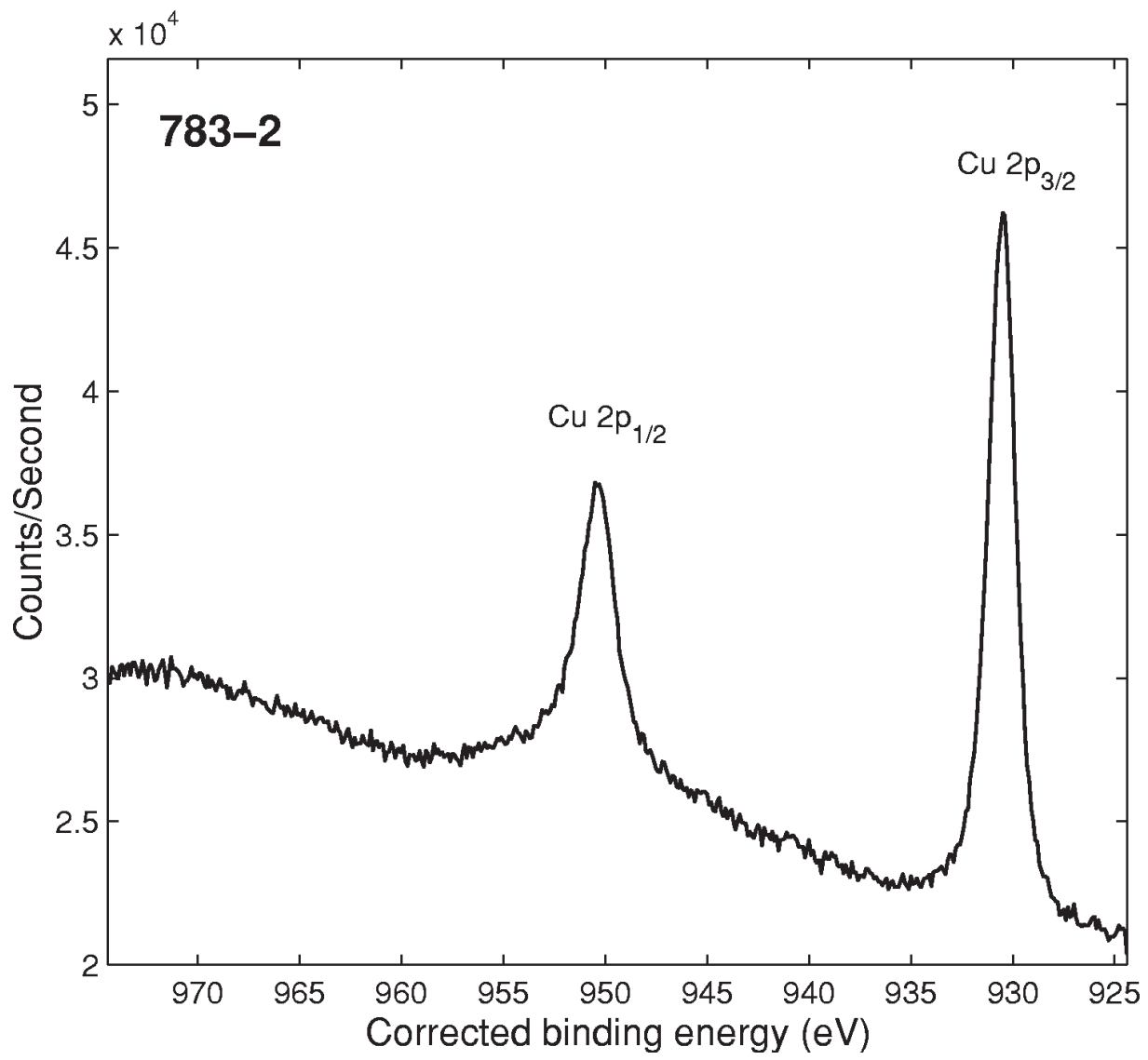

- Accession \#: 00783-02

- Host Material: Synthetic enargite

- Technique: XPS

- Spectral Region: Cu $2 p$

Instrument: Vacuum Generators Ltd., East Greenstead, UK Escalab Mark II with upgrade to Escalab 200

Excitation Source: $\mathrm{Al} K_{\alpha}$ Source Energy: $1486.6 \mathrm{eV}$ Source Strength: $300 \mathrm{~W}$ Source Size: $12.5 \mathrm{~mm} \times 12.5 \mathrm{~mm}$ Incident Angle: $49^{\circ}$ Analyzer Type: spherical sector Analyzer Pass Energy: $20 \mathrm{eV}$ Analyzer Resolution: $1.12 \mathrm{eV}$ Emission Angle: $0^{\circ}$ Total Signal Accumulation Time: $90.10 \mathrm{~s}$

Total Elapsed Time: $90.10 \mathrm{~s}$

Number of Scans: 1

Effective Detector Width: $0.4 \mathrm{eV}$

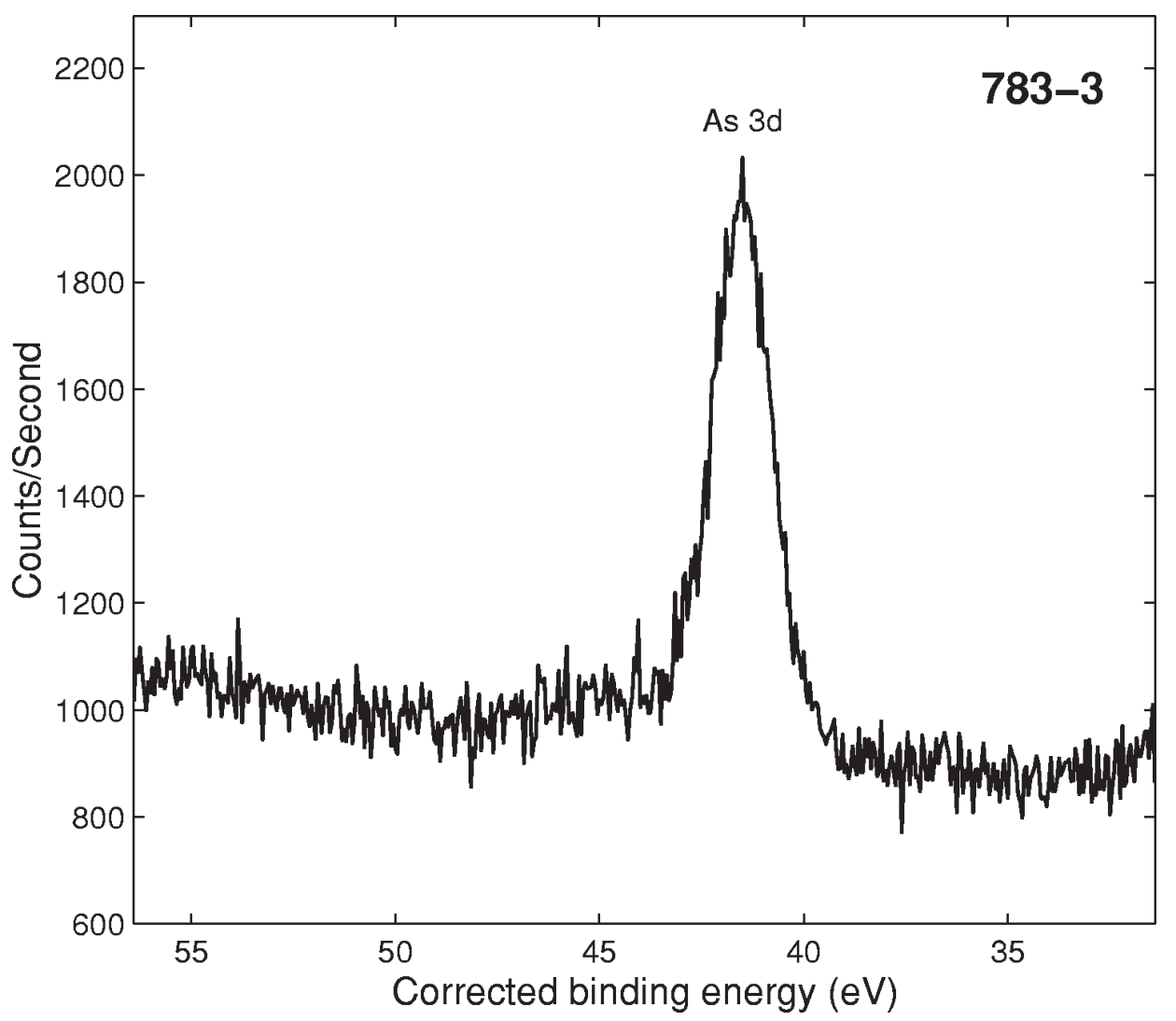

Accession \#: 00783-03

- Host Material: Synthetic enargite

- Technique: XPS

- Spectral Region: As $3 d$

Instrument: Vacuum Generators Ltd., East Greenstead, UK Escalab Mark II with upgrade to Escalab 200

Excitation Source: Al $\mathrm{K}$ Source Energy: $1486.6 \mathrm{eV}$ Source Strength: $300 \mathrm{~W}$ Source Size: $12.5 \mathrm{~mm} \times 12.5 \mathrm{~mm}$ Incident Angle: $49^{\circ}$ Analyzer Type: spherical sector Analyzer Pass Energy: $20 \mathrm{eV}$ Analyzer Resolution: $1.12 \mathrm{eV}$ Emission Angle: $0^{\circ}$

Total Signal Accumulation Time: $150.30 \mathrm{~s}$

Total Elapsed Time: $150.30 \mathrm{~s}$

Number of Scans: 3

Effective Detector Width: $0.4 \mathrm{eV}$ 


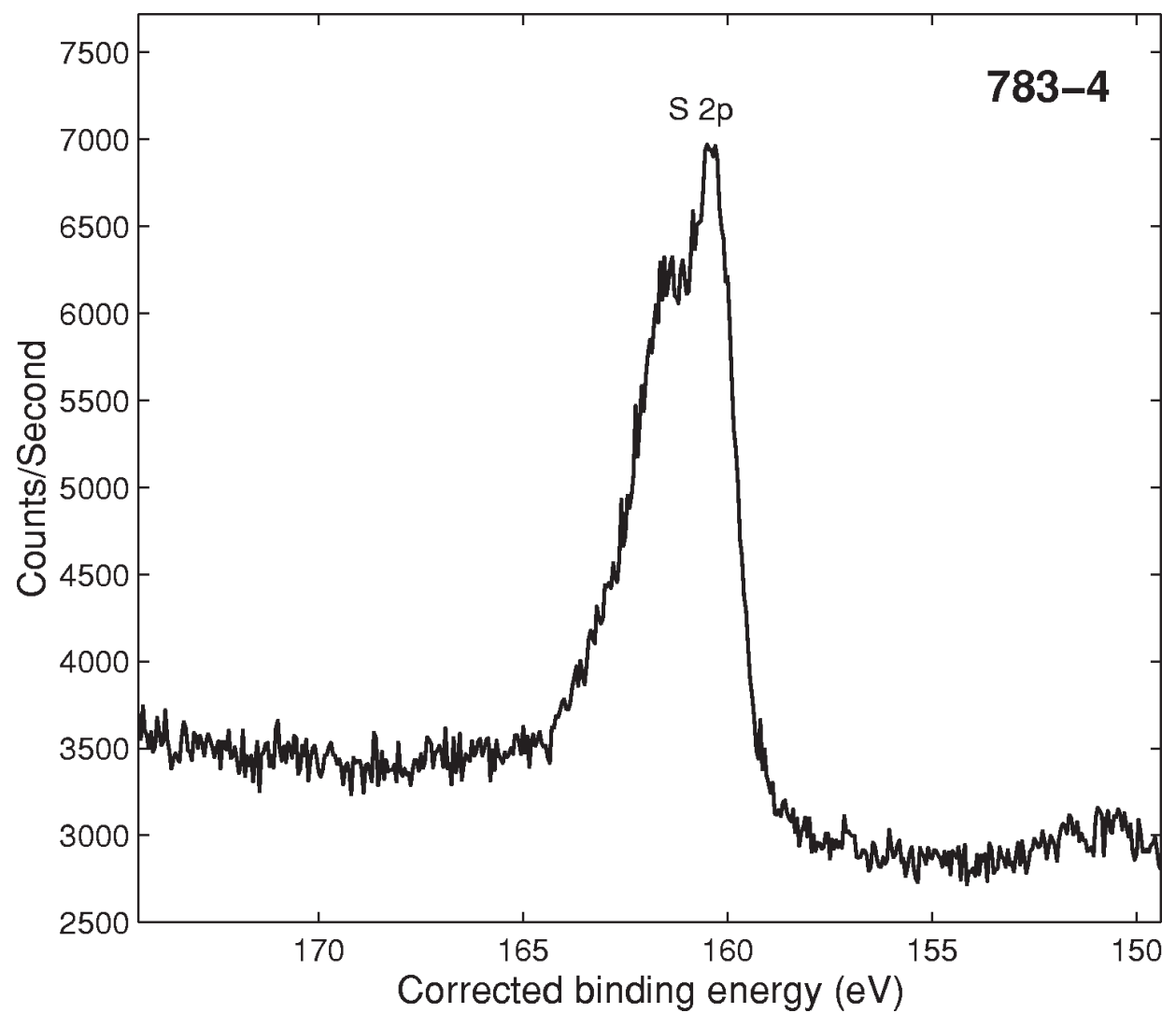

- Accession \#: 00783-04

nost Material: Synthetic enargite

- Technique: XPS

- Spectral Region: $S 2 p$

Instrument: Vacuum Generators Ltd., East Greenstead, UK Escalab Mark II with upgrade to Escalab 200

Excitation Source: Al $K_{\alpha}$ Source Energy: $1486.6 \mathrm{eV}$ Source Strength: $300 \mathrm{~W}$

Source Size: $12.5 \mathrm{~mm} \times 12.5 \mathrm{~mm}$ Incident Angle: $49^{\circ}$ Analyzer Type: spherical sector Analyzer Pass Energy: $20 \mathrm{eV}$ Analyzer Resolution: $1.12 \mathrm{eV}$ Emission Angle: $0^{\circ}$

Total Signal Accumulation Time: $150.30 \mathrm{~s}$

Total Elapsed Time: $150.30 \mathrm{~s}$

Number of Scans: 3

Effective Detector Width: $0.4 \mathrm{eV}$

Comment: See footnote below the Spectral Features Table. 